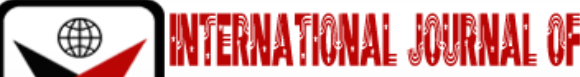

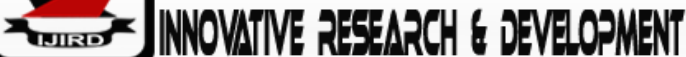

ISSN 2278-0211 (Online)

\section{Haematological and Histological Response of Clarias Gariepinus (Burchell, 1822) Fed Varied Inclusion Level of Defatted Groundnut Cake in Plant Protein Based Diet}

\author{
Jacob Oyeleye Oyedokun \\ Lecturer, Department of Fisheries and Aquaculture, \\ Adekunle Ajasin University, Akungba-Akoko, Ondo State
}

\begin{abstract}
:
This Study Was Aimed At Determining The Haematological And Histological Effects Of Feeding Clarias Gariepinus (C. Gariepinus) Juveniles With Plant Protein-Based Diets With Varying Inclusion Of Defatted Groundnut Cake (DGNC). Fingerling's Fish (N=360), Weighing 6.88 $\pm 0.20 \mathrm{~g}$ Were Randomly Allocated To Six Diets Containing Varied Inclusion Of Defatted Groundnut Cake In The Formulated Basal Plant Protein-Based Diet With 0\% (Control), 20\% (DGNC 20\%), 40\% (DGNC 40\%), 60\% (DGNC 60\%), 80\% (DGNC 80\%) And 100\% (DGNC 100\%) Inclusion Of Defatted Groundnut Cake. The Experiment Lasted For Eight Weeks. Significantly (P>0.05) Higher Weight Gain Value Of 143.61 $\pm 94.91 \mathrm{~g}$ Was Observed In DGNC 20\%. Feed Conversion Ratio Had The Least (P>0.05) Value Of 1.78 $\pm 0.85 \mathrm{~g}$ In DGNC 20\% Compare To Other Treatments. C. Gariepinus Had A Significantly Higher PVC Values Of 25.00\% In Fish On DGNC 40\% And Least In Control Diet. Fish Fed DGNC 40\% Had A Significantly Higher WBC Values Of 57200 $26972.39 \mathrm{~mm}$. Significantly Higher RBC $(1.92 \pm 0.62 \times 1012 / L)$ Was Observed In Fish Fed DGNC 40\% And The Least Value $(0.62 \pm 0.13 \times 1012 / L)$ In DGNC 100\%. Histological Examinations Of Fish Organs Like Liver, Gill And Kidney Showed Some Toxic Effects. These Toxic Effects Were Varied In Different Organs Ranging From Mild Lesions To More Marked Ones. Organs Of Fish Fed Above 20\% Inclusion Level Of DGNC Were Mostly Affected. However, Defatted Groundnut Cake Has Good Potential As Feed Ingredient In The Substitution Of Soyabean Meal In The Diet Of C. Gariepinus. Substitution Of Defatted Groundnut Cake As A Replacement For Soyabean Meal Beyond 20\% May Lead To Serious Nutritional And Health Hazard Of The Fish.
\end{abstract}

Keywords: Soyabean, defatted groundnut cake, heamatological, histology, clarias gariepinus

\section{Introduction}

Aquaculture has become the fastest growing food production sector of the world, with an average annual increase of about 10\% in 2000 compared to 3\% increase for livestock meat and 1.6\% increase in capture fisheries (FA0, 2000). One of the most promising enterprises in the aquaculture business in Nigeria is the catfish (Clarias gariepinus) farming and this is due to their fast growth, tolerance etc.

Clarias gariepinus (C. gariepinus) is one of the best aquaculture species that is suitable for culture in tropical clime because of its possession of certain attributes. C. gariepinus is an esteemed fish food that has high dressing percentage and good consumer acceptance than other cultured freshwater species. Fast growth and enhanced production of this economic fish is presumed to be directly related to the quality of its feed (Olapade and George, 2019).

Consequently, one of the essential prerequisites for the successful management of this fish culture programme is a comprehensive understanding of feeding (Halver, 1972). In Aquaculture, fish feed is conventionally formulated to contain high amount of fishmeal (32-40\%) (Richard and Chapman, 2007). Fish meal which contains the essential amino acids needed by fish in the diet is very expensive thus increasing the cost of fish production. This has compelled fish culturists to constantly consider and explore alternative protein-rich dietary supplements that are cheap, locally available and nutritionally safe for use as fishmeal replacers in fish diets. Partial replacement of fishmeal in the diet of different cultured fish species with plant-based protein ingredient sources has been achieved. Complete replacement of fishmeal by plant protein sources has however not been achieved due to certain limitations inherent in them.

Similarly, soybean meal has always been the principal plant protein source used in fish feeds as a replacement for fishmeal owing to its high protein content and relatively well-balanced amino acid profile (Sintayehu et al., 1996). However, soybean meal has been increasingly commercialized and variously used in human, livestock and poultry dietary formulations, hence its extensive utilization as the main protein source in fish feeds may no longer be economically viable (Siddhuraju and Becker, 2001). Therefore, less competitive and locally available plant protein sources such as groundnut cake meal should be considered as an alternative to replace soybean meal in plant-based diet without reducing the nutritional quality of the feed. 
Groundnut cake is an alternative protein which is of plant source and many authors in different studies have used Groundnut Cake Meal (GNC) as a suitable plant protein source to substitute for fishmeal. The GNC is reported to be highly palatable with better binding properties than soyabean meal (Olapade and George, 2019). It is however, deficient in some vital amino acids (such as lysine and methionine) that are present in fishmeal (Eyo and Olatunde, 1998). Its amino acid quality improves in artificial diets when reinforced with lysine and methionine (Davies and Ezenwa, 2010; Oyedokun et al., 2019).

However, for a plant protein ingredient to be included in fish feed, its utilization should be tested in different fish species which differ in their sensitivity and response to anti-nutrients present in such plant protein sources (Chaudhuri et al., 2012). Adeparusi and Ajayi (2004) reported that blood analysis is an important factor that could be considered in fish feed assessment. Haematological profile has been used as a tool to evaluate fish welfare and disturbance on fish metabolism (Barros et al., 2009; Barbieri and Bondioli, 2015). Similarly, Histopathological changes have been widely used as biomarkers in the assessment of fish health status after their exposure to various chemicals/contaminants/ feeding trials in the laboratory (Thophon et al., 2003). Few studies on the effects of replacing soyabean meal with groundnut cake meal in a plant protein-based diet on the blood profile and organs of C. gariepinus are limited. Therefore, the project is aimed at assessing the growth performance, blood profile and organs of C. gariepinusfed varied inclusion of DGNC in a plant-based diet.

\section{Materials and Method}

This study was carried out at the Fisheries and Aquaculture Laboratory, located at the Central Laboratory of Adekunle Ajasin University, Akungba-Akoko, Ondo State. Iso- nitrogenous basal diet in each plant protein diet with varying inclusion levels of DGNC to replace soyabean meal was used to formulate six (6) dietary treatment (0\%, 20\%, 40\%, 60\%, $80 \%$ and $100 \%$ inclusion levels of DGNC). The diets were formulated to $40 \%$ crude protein (Faturoti et al.,1986). Gross composition of experimental diets is shown in Table 1. C. gariepinus juveniles $(\mathrm{n}=360)$ aged two months weighing 136.4 $\pm 2.53 \mathrm{~g}$ were acquired from Hatchery unit, Sanni Farms, Ikare Akoko. The fish were acclimatized under laboratory condition for two weeks and fed $2 \mathrm{~mm}$ skretting diet. Healthy fish were randomly distributed into eighteen plastic tanks of 25L capacity each at a stocking density of twenty (20) fish per tank. Water temperature was kept at optimum range of 27.41 and 29.62oC. The fish were left for 2 days unfed before the experiment. The fish were fed to satiation twice daily (08:00 and17:00 hours). The weight of the fish was monitored weekly. Water quality was monitored weekly. The experiment lasted for 70 days.

\subsection{Experimental Diets}

Feedstuffs for the research work include Soyabeans, Groundnut cake meal. Methionine, Lysine, Maize, Fish oil, Salt, Vitamin C, DCP, Calcium carbonate (CaCo3), Chromium oxide, Vitamin premix. The six experimental diets were formulated to contain $85 \%$ inclusion level of soyabean meal and varying percentage inclusion of defatted groundnut cake meal in the following inclusion percentage of $0 \%, 20 \%, 40 \%, 60 \%, 80 \%$, and $100 \%$ which contains $40 \%$ crude protein. The defatted groundnut used in the diet was obtained from a local market alongside the soyabean meal and the other ingredient. All the ingredient was thoroughly mixed together manually using clean borehole water and subsequently pelleted using a floating pelleting machine. The feed was pelletized using a $3 \mathrm{~mm}$ pelletizer. The pelleted diets were sun dried and stored in a labelled air tight container.

\begin{tabular}{|c|c|c|c|c|c|c|}
\hline $\begin{array}{c}\text { INGREDIENTS } \\
\text { (g/100g) }\end{array}$ & $\begin{array}{c}\text { DGNC } \\
0 \% \\
\end{array}$ & $\begin{array}{c}\text { DGNC } \\
20 \% \\
\end{array}$ & $\begin{array}{c}\text { DGNC } \\
40 \% \\
\end{array}$ & $\begin{array}{c}\text { DGNC } \\
60 \% \\
\end{array}$ & $\begin{array}{c}\text { DGNC } \\
\mathbf{8 0 \%} \\
\end{array}$ & $\begin{array}{l}\text { DGNC } \\
100 \% \\
\end{array}$ \\
\hline Soyabeans & 85.8 & 68 & 51 & 34 & 17 & - \\
\hline Groundnut cake meal & - & 17.8 & 34.8 & 51.8 & 68.8 & 85.8 \\
\hline Methionine & 0.4 & 0.4 & 0.4 & 0.4 & 0.4 & 0.4 \\
\hline Lysine & 0.6 & 0.6 & 0.6 & 0.6 & 0.6 & 0.6 \\
\hline Maize & 10 & 10 & 10 & 10 & 10 & 10 \\
\hline Fish oil & 1 & 1 & 1 & 1 & 1 & 1 \\
\hline Salt & 0.1 & 0.1 & 0.1 & 0.1 & 0.1 & 0.1 \\
\hline Vitamin c & 0.2 & 0.2 & 0.2 & 0.2 & 0.2 & 0.2 \\
\hline DCP & 1 & 1 & 1 & 1 & 1 & 1 \\
\hline $\begin{array}{c}\text { Calcium } \\
\text { carbonate }\left(\mathrm{CaCO}_{3}\right)\end{array}$ & 0.4 & 0.4 & 0.4 & 0.4 & 0.4 & 0.4 \\
\hline${ }^{*}$ Vitamin premix & 0.5 & 0.5 & 0.5 & 0.5 & 0.5 & 0.5 \\
\hline Total & 100 & 100 & 100 & 100 & 100 & 100 \\
\hline
\end{tabular}

Table 1: Ingredient Composition of Experimental Diet (DGNC)

*Vitamin premix contains/kg: vitamins. A: 4 x107 i.u, D3: 6x106 i.u, K3: 800mg; Tocophenols, 2x105i.u, Folacin $100 \mathrm{mg}$, Thiame $200 \mathrm{mg}$, Riboflavin $6000 \mathrm{mg}$, Niacin $4 \times 105$, Calcium panththenate $10000 \mathrm{mg}$, Pyridoxine $3000 \mathrm{mg}$, Cyanocobalamin 12mg, Biotin $80 \mathrm{mg}$, Mn $6000 \mathrm{mg}$, Zn $8000 \mathrm{mg}$, Fe $8000 \mathrm{mg}$, Cu $800 \mathrm{mg}$, Choline chloride $8 \times 105 \mathrm{mg}$, iodine 1`03mg, Co 4x103mg, Se 2x103mg BHT 2x10mg 


\subsection{Proximate Analysis of Feed Stuffs and Ingredient}

The proximate analysis was carried out at the Animal Nutrition and Biochemistry Laboratory of the Department of Animal Science, University of Ibadan based on the standard of association of analytical chemist (AOAC, 2006).

\begin{tabular}{|c|c|c|c|c|c|c|}
\hline $\begin{array}{c}\text { Treatment } \\
\text { \% }\end{array}$ & DGNC 0\% & $\begin{array}{c}\text { DNGC } \\
\text { \% }\end{array}$ & DGNC 40\% & DGNC 60\% & DGNC 80\% & DGNC 100\% \\
\hline $\begin{array}{c}\text { Crude } \\
\text { Protein }\end{array}$ & $28.84 \pm 0.27^{\mathrm{f}}$ & $35.95 \pm 0.15^{\mathrm{c}}$ & $36.47 \pm 0.05^{\mathrm{b}}$ & $39.03 \pm 0.24^{\mathrm{a}}$ & $34.63 \pm 0.04^{\mathrm{d}}$ & $34.33 \pm 0.03^{\mathrm{e}}$ \\
\hline $\begin{array}{c}\text { Ether } \\
\text { Extract }\end{array}$ & $6.65 \pm 0.15^{\mathrm{b}}$ & $7.00 \pm 0.10^{\mathrm{a}}$ & $7.15 \pm 0.05^{\mathrm{a}}$ & $6.70 \pm 0.10^{\mathrm{b}}$ & $6.60 \pm 0.10^{\mathrm{b}}$ & $6.35 \pm 0.05^{\mathrm{c}}$ \\
\hline Ash Content & $7.50 \pm 0.10^{\mathrm{a}}$ & $6.80 \pm 0.10^{\mathrm{c}}$ & $7.35 \pm 0.15^{\mathrm{ab}}$ & $6.45 \pm 0.15^{\mathrm{d}}$ & $6.65 \pm 0.05 \mathrm{c}^{\mathrm{d}}$ & $7.27 \pm 0.14^{\mathrm{b}}$ \\
\hline Crude Fiber & $3.87 \pm 0.04^{\mathrm{a}}$ & $3.63 \pm 0.03^{\mathrm{b}}$ & $3.53 \pm 0.03^{\mathrm{b}}$ & $3.30 \pm 0.10^{\mathrm{c}}$ & $3.50 \pm 0.10^{\mathrm{b}}$ & $3.60 \pm 0.10^{\mathrm{b}}$ \\
\hline Dry Matter & $90.96 \pm 0.06^{\mathrm{d}}$ & $91.34 \pm 0.02^{\mathrm{b}}$ & $91.29 \pm 0.01^{\mathrm{b}}$ & $91.91 \pm 0.05^{\mathrm{a}}$ & $90.68 \pm 0.02^{\mathrm{e}}$ & $91.19 \pm 0.04^{\mathrm{c}}$ \\
\hline \multicolumn{7}{|c|}{ Table 2: Proximate Composition of Feedstuffs }
\end{tabular}

$a, b, c, d, f=$ Indicate That Means on the Same Row But with Different Superscripts Are Statistically Significant (P<0.05)

\subsection{Growth and Nutrient Utilization Parameters Analysis}

The following growth and nutrient utilization parameter were calculated as described by Stickey (2006). Mean Weight Gain (MWG), Feed Intake (FI), Specific Growth Rate (SGR) Protein Efficiency Ratio (PER), Feed Conversion Ratio (FCR), Feed efficiency ratio (FER), Mean weight gain (MWG), Survival rate and Gross Efficiency of Feed Conversion (GEFC).

\subsection{Blood Sampling and Analysis}

The fishes were randomly selected with a small hand net and placed belly upward. Blood samples of about 4 milliliters was collected from the caudal peduncle (Stoskopf, 1993) with the aid of a $2 \mathrm{~cm} 3$ syringe,1ml of the blood was dispensed into ethylene diamine tetra-acetic acid (EDTA) anticoagulant for haematological studies. The blood samples were analysed for packed cell volume (PCV), haemoglobin concentration, red blood cell (RBC), white blood cell (WBC) described by Dacie and Lewis (1991) and Joshi et al, (2002).

\subsubsection{Mean Corpuscular Volume (MCV)}

MCV was estimated using the model described by Dacie and Lewis (1991):

$\mathrm{MCV}=\frac{\text { volume of red blood cell (in } \mathrm{mL} \text { per } 100 \mathrm{~mL} \text { blood) }}{\text { Number of red blood cells oer } 100 \mathrm{~mL} \text { blood }} \times 100$

\subsubsection{Mean Corpuscular Haemoglobin (MCH)}

MCH was estimated using the model described by Stoskopf, 1993.

$\mathrm{MCH}=\frac{\text { Haemoglobin }\left(\frac{g}{100 \mathrm{ml}}\right)}{\text { Number of red blood cells }\left(\frac{\mathrm{millions}}{L} \text { blood }\right)} \times 100$

\subsubsection{Mean Corpuscular Haemoglobin Concentration (MCHC)}

MCHC was estimated using the model as described by Stoskopf, 1993

MCHC $=\frac{\text { Haemoglobin } \text { concentration }}{\text { Packed cell volume }} \times 100$

\subsection{Histological Analysis}

The histopathological examinations were carried out on the gill, liver and kidney of the fish at the Department of Veterinary Pathology, University of Ibadan. Fish organs such as liver, gills and kidney from each experimental unit were removed and examined for possible changes due to the treatment effects on the fish. One fish was sacrificed from each replicate (i.e., three fish per treatment) and selected organs promptly removed, the organs were carefully removed from the internal organs of the fish, so as to avoid damage and preserved in $10 \%$ formalin solution. The fixed tissues were processed routinely for histological analysis as described by Samuelson (2007). The necrotized areas were then photographed and read accordingly to determine the histopathological effects of varying inclusion of groundnut cake in a plant protein-based diet.

\subsection{Statistical Analysis of Data}

The data collected were subjected to one-way analysis of variance (ANOVA). Where the ANOVA reveals significant difference $(\mathrm{p}<0.05)$ and Duncan multiple range test was used to compare differences among individual treatment means.

\section{Results}

There was no significant difference $(\mathrm{P}>0.05)$ for the values of initial mean weight, specific growth rate, survival rate, protein efficiency ratio and gross efficiency feed conversion for all the treatments (Table 3). Significantly higher value $(\mathrm{P}>0.05)$ was observed in C. gariepinus fed diet DGNC 20\% (16.48 $\pm 6.55 \mathrm{~g}, 9.85 \pm 6.42 \mathrm{~g}, 143.61 \pm 94.91 \mathrm{~g})$ and least in DGNC $100 \%(8.60 \pm 1.90 \mathrm{~g}, 1.69 \pm 1.88 \mathrm{~g}, 24.43 \pm 27.17 \mathrm{~g})$ in final mean weight, mean weight gain and weight gain respectively. However, other treatments were intermediate to them. Significant difference $(\mathrm{P}>0.05)$ was observed in feed conversion ratio with the least values in DGNC 20\% (1.74 $\pm 0.85 \mathrm{~g})$ and the highest value in DGNC $100 \%(2.93 \pm 0.33 \mathrm{~g})$. Significant 
increases ( $P>0.05)$ were observed in DGNC 20\% (0.68 $\pm 0.35 \mathrm{~g})$ and decreases in DGNC 100\% $(0.34 \pm 0.35 \mathrm{~g})$ for feed efficiency ratio. However, the other is intermediate. Protein intake values ranges from $2.07 \pm 0.15 \mathrm{~g}$ in $100 \%$ DGNC to $1.36 \pm 0.12 \mathrm{~g}$ in $20 \%$ DGNC. However, control diet is similar to $100 \%$ DGNC and $40 \%, 60 \%$ and $80 \%$ DGNC are similar to $100 \%$ DGNC. Significantly higher ( $>0.05)$ values were observed in C. gariepinus fed diet containing DGNC $100 \%$ $(5.17 \pm 0.37 \mathrm{~g})$ followed by control diet $(4.78 \pm 0.28 \mathrm{~g})$, DGNC $80 \%(3.69 \pm 0.13 \mathrm{~g})$, DGNC $60 \%(3.66 \pm 0.45 \mathrm{~g})$, DGNC $40 \%$ $(3.65 \pm 0.17 \mathrm{~g})$ and DGNC $20 \%(3.40 \pm 0.29 \mathrm{~g})$.

\begin{tabular}{|c|c|c|c|c|c|c|}
\hline $\begin{array}{c}\text { Parameters } \\
\mathbf{( g )}\end{array}$ & $\begin{array}{c}\text { DGNC } \\
\mathbf{0 \%}\end{array}$ & $\begin{array}{c}\text { DGNC } \\
\mathbf{2 0 \%}\end{array}$ & $\begin{array}{c}\text { DGNC } \\
\mathbf{4 0 \%}\end{array}$ & $\begin{array}{c}\text { DGNC } \\
\mathbf{6 0 \%}\end{array}$ & $\begin{array}{c}\text { DGNC } \\
\mathbf{8 0 \%}\end{array}$ & $\begin{array}{c}\text { DGNC } \\
\mathbf{1 0 0 \%}\end{array}$ \\
\hline $\begin{array}{c}\text { Initial Mean } \\
\text { Weight }\end{array}$ & $6.91 \pm 0.00^{\mathrm{a}}$ & $6.88 \pm 0.06^{\mathrm{a}}$ & $6.85 \pm 0.04^{\mathrm{a}}$ & $6.89 \pm 0.03^{\mathrm{a}}$ & $6.91 \pm 0.04^{\mathrm{a}}$ & $6.91 \pm 0.02^{\mathrm{a}}$ \\
\hline $\begin{array}{c}\text { Final Mean } \\
\text { Weight }\end{array}$ & $12.18 \pm 0.29^{\mathrm{ab}}$ & $16.48 \pm 6.56^{\mathrm{a}}$ & $14.90 \pm 1.92^{\mathrm{ab}}$ & $12.48 \pm 1.30^{\mathrm{ab}}$ & $13.09 \pm 2.19^{\mathrm{ab}}$ & $8.60 \pm 1.90^{\mathrm{b}}$ \\
\hline $\begin{array}{c}\text { Mean } \\
\text { Weight Gain }\end{array}$ & $5.27 \pm 0.29^{\mathrm{ab}}$ & $9.85 \pm 6.42^{\mathrm{a}}$ & $8.05 \pm 1.96^{\mathrm{ab}}$ & $5.60 \pm 1.28^{\mathrm{ab}}$ & $6.18 \pm 2.16^{\mathrm{ab}}$ & $1.69 \pm 1.88^{\mathrm{b}}$ \\
\hline WG & $76.19 \pm 4.20^{\mathrm{ab}}$ & $143.61 \pm 94.91^{\mathrm{a}}$ & $117.50 \pm 29.16^{\mathrm{ab}}$ & $81.23 \pm 18.27^{\mathrm{ab}}$ & $89.38 \pm 30.65^{\mathrm{ab}}$ & $24.43 \pm 27.17^{\mathrm{b}}$ \\
\hline FCR & $1.96 \pm 0.24^{\mathrm{ab}}$ & $1.74 \pm 0.85^{\mathrm{b}}$ & $1.78 \pm 0.30^{\mathrm{ab}}$ & $2.07 \pm 0.50^{\mathrm{ab}}$ & $2.72 \pm 0.95^{\mathrm{ab}}$ & $2.93 \pm 0.33^{\mathrm{a}}$ \\
\hline GEFC & $52.45 \pm 9.01^{\mathrm{a}}$ & $68.65 \pm 35.44^{\mathrm{a}}$ & $57.30 \pm 9.14^{\mathrm{a}}$ & $49.99 \pm 10.94^{\mathrm{a}}$ & $40.45 \pm 16.25^{\mathrm{a}}$ & $32.59 \pm 7.88^{\mathrm{a}}$ \\
\hline Feed Intake & $4.78 \pm 0.28^{\mathrm{a}}$ & $3.40 \pm 0.29^{\mathrm{b}}$ & $3.65 \pm 0.17^{\mathrm{b}}$ & $3.66 \pm 0.45^{\mathrm{b}}$ & $3.69 \pm 0.13^{\mathrm{b}}$ & $5.17 \pm 0.37^{\mathrm{a}}$ \\
\hline $\begin{array}{c}\text { Protein } \\
\text { Intake }\end{array}$ & $1.91 \pm 0.11^{\mathrm{a}}$ & $1.36 \pm 0.12^{\mathrm{b}}$ & $1.46 \pm 0.07^{\mathrm{b}}$ & $1.46 \pm 0.18^{\mathrm{b}}$ & $1.48 \pm 0.05^{\mathrm{b}}$ & $2.07 \pm 0.15^{\mathrm{a}}$ \\
\hline FER & $0.52 \pm 0.07^{\mathrm{ab}}$ & $0.68 \pm 0.35^{\mathrm{a}}$ & $0.57 \pm 0.09^{\mathrm{ab}}$ & $0.50 \pm 0.11^{\mathrm{ab}}$ & $0.41 \pm 0.16^{\mathrm{ab}}$ & $0.34 \pm 0.35^{\mathrm{b}}$ \\
\hline PER & $2.48 \pm 0.05^{\mathrm{a}}$ & $3.48 \pm 1.87^{\mathrm{a}}$ & $3.11 \pm 0.27^{\mathrm{a}}$ & $2.58 \pm 0.46^{\mathrm{a}}$ & $2.21 \pm 0.83^{\mathrm{a}}$ & $1.96 \pm 0.64^{\mathrm{a}}$ \\
\hline SGR & $9.28 \pm 0.05^{\mathrm{a}}$ & $9.80 \pm 1.07^{\mathrm{a}}$ & $9.75 \pm 0.18^{\mathrm{a}}$ & $9.34 \pm 0.39^{\mathrm{a}}$ & $8.93 \pm 0.77^{\mathrm{a}}$ & $8.55 \pm 0.76^{\mathrm{a}}$ \\
\hline $\begin{array}{c}\text { Survival } \\
\text { Rate (\%) }\end{array}$ & $97.50 \pm 3.54^{\mathrm{a}}$ & $86.67 \pm 10.41^{\mathrm{a}}$ & $88.33 \pm 7.64^{\mathrm{a}}$ & $96.67 \pm 2.89^{\mathrm{a}}$ & $86.67 \pm 2.89^{\mathrm{a}}$ & $95.00 \pm 0.00^{\mathrm{a}}$ \\
\hline
\end{tabular}

Table 3: Growth and Nutrient Utilization of Clarias Gariepinus Fed DGNC Based Diets

$a, b, c, d=$ Indicate That Means on the Same Row But with Different Superscripts Are Statistically Significant $(P<0.05)$ $S G R=$ Specific Growth Rate, FCR = Feed Conversion Ratio, PER = Protein Efficiency Ratio, FER $=$ Feed Efficiency Ratio, GEFC $=$ Gross Efficiency Of Feed Conversion, $W G=$ Weight Gain.

There were no significant differences $(\mathrm{P}<0.05)$ in the values of PLATELET, MCHC, MVC and EOSIN for all the treatments (Table 4). Significantly higher values were observed in diet containing 40\% DGNC $(25.00 \pm 6.56 \%)$ and least value in diet containing $100 \%$ DGNC $(9.00 \pm 1.41 \%)$ for the PCV. While the others are intermediate. Significant difference $(\mathrm{P}<0.05)$ was observed in WBC with the least value in the control diet $(8550 \pm 869.74(104 / \mathrm{mm} 3))$ and the highest value in diet containing $40 \%$ DGNC $(57200 \pm 26972.39(104 / \mathrm{mm} 3))$. A significant increase was observed for the values of the NEUTROPHIL at $100 \%$ inclusion of DGNC $(5.50 \pm 2.12 \%)$ and reduction at $40 \%$ inclusion of DGNC $(1.65 \pm 0.58 \%)$, the other treatments are intermediate. There was significant difference $(\mathrm{P}<0.05)$ in the LYMPHOCYTE values for all the treatments. The highest value $96.67 \pm 1.53$ was observed in $20 \%$ inclusion level of DGNC and the lowest value $85.00 \pm 0.00$ in $100 \%$ inclusion level of DGNC. Diet containing $100 \%$ DGNC (7.50 \pm 2.12$)(1.00 \pm 0.00)$ was significantly difference from the other treatments for MONOCYCTE and BASOPHIL respectively. HAEMOGLOBIN values significantly range from $8.56 \pm 2.16$ for $40 \%$ inclusion level to $3.15 \pm 0.64$ for $100 \%$ inclusion level of DGNC. However, the control diet was similar to $100 \%$ inclusion level of DGNC. The RBC counts observed a significant increase in $40 \%$ inclusion level of DGNC $(1.97 \pm 0.62)$ and a significant decrease in $100 \%$ inclusion level of DGNC $(0.62 \pm 0.13)$. However, the other treatments are intermediate. $\mathrm{MCH}$ values significantly ranges from $55.00 \pm 10.47$ for control diet to $30.07 \pm 20.51$ for diet containing $20 \%$ DGNC. However, diet containing $80 \%$ DGNC is similar to control diet. The other diets are intermediate. 


\begin{tabular}{|c|c|c|c|c|c|c|}
\hline Parameter & $\begin{array}{c}\text { DGNC } \\
\mathbf{0} \% \\
\end{array}$ & $\begin{array}{c}\text { DGNC } \\
20 \% \\
\end{array}$ & $\begin{array}{c}\text { DGNC } \\
40 \% \\
\end{array}$ & $\begin{array}{c}\text { DGNC } \\
60 \% \\
\end{array}$ & $\begin{array}{c}\text { DGNC } \\
80 \%\end{array}$ & $\begin{array}{l}\text { DGNC } \\
100 \% \\
\end{array}$ \\
\hline 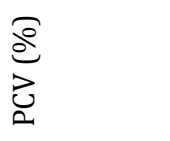 & 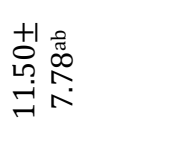 & 岱 & 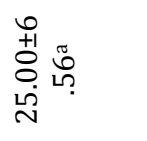 & 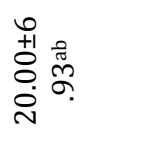 & 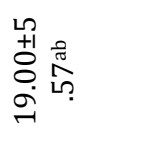 & 市苛 \\
\hline 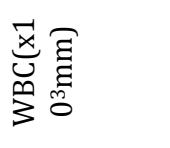 & 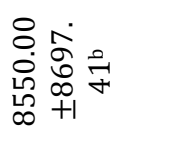 & 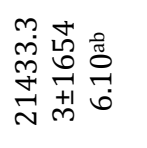 & 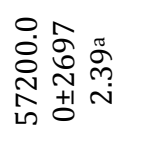 & 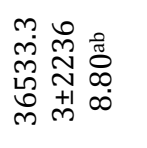 & 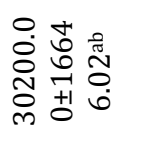 & 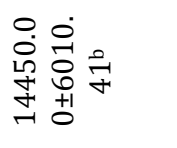 \\
\hline 言 & 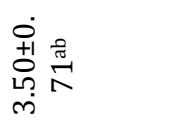 & 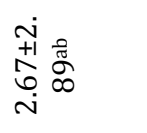 & 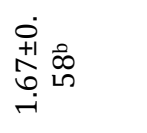 & 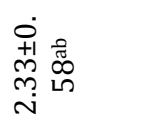 & 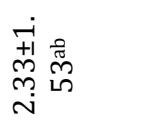 & 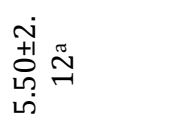 \\
\hline 胥す & $\begin{array}{l}+1 \\
\stackrel{1}{0} \\
\dot{8} \\
8 \\
8\end{array}$ & 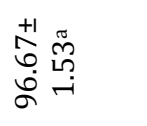 & 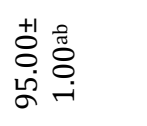 & 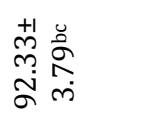 & 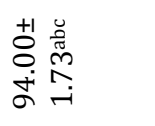 & 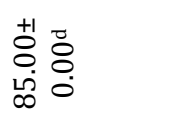 \\
\hline $\begin{array}{l}\text { a } \\
0 \\
0 \\
\vdots \\
\sum\end{array}$ & 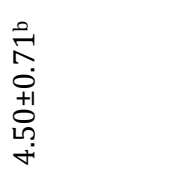 & $\begin{array}{l}\dot{8} \\
\dot{8} \\
\dot{+} \\
\dot{+1} \\
\dot{0} \\
\dot{m}\end{array}$ & 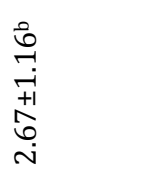 & $\begin{array}{l}\stackrel{0}{\infty} \\
\stackrel{1}{j} \\
+1 \\
+1 \\
\dot{6} \\
\dot{m}\end{array}$ & 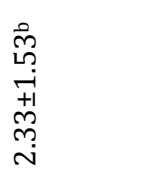 & 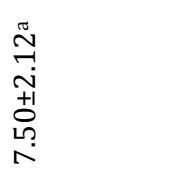 \\
\hline 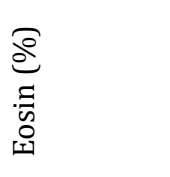 & 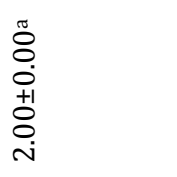 & 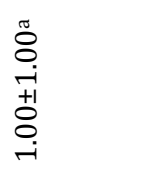 & 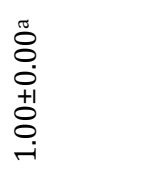 & 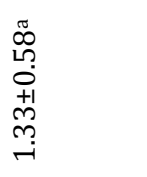 & $\begin{array}{l}\text { ó } \\
0 \\
0 \\
+1 \\
0 \\
0 \\
-\end{array}$ & $\begin{array}{l}0 \\
0 \\
0 \\
0 \\
+1 \\
0 \\
0 \\
-1\end{array}$ \\
\hline 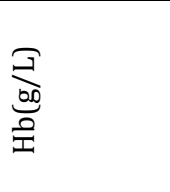 & 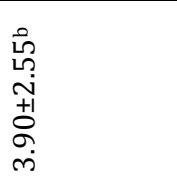 & 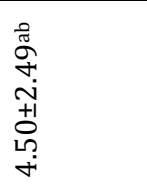 & 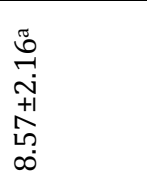 & 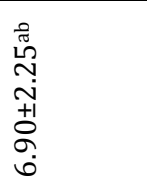 & 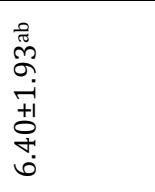 & 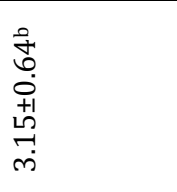 \\
\hline 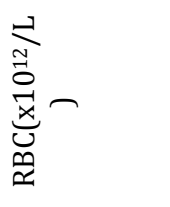 & $\begin{array}{l}\stackrel{u}{0} \\
\text { Ln } \\
\\
0 \\
+1 \\
0 \\
0 \\
0 \\
0\end{array}$ & 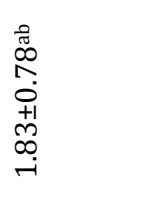 & 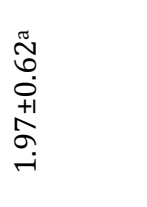 & 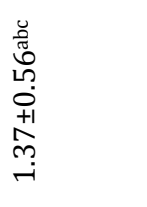 & 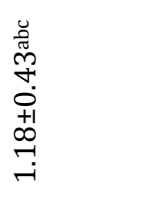 & 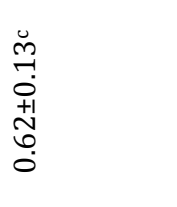 \\
\hline $\begin{array}{l}\Xi \\
\Xi \\
\dot{U}\end{array}$ & 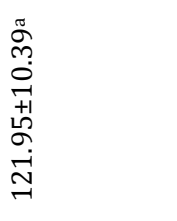 & 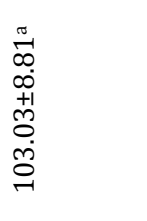 & 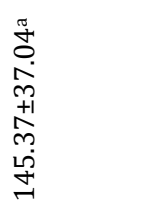 & 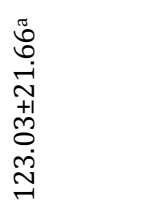 & 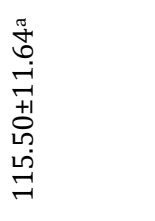 & 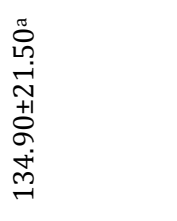 \\
\hline 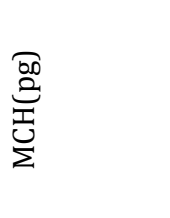 & 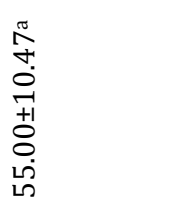 & 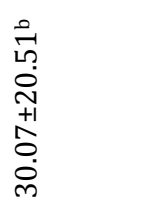 & 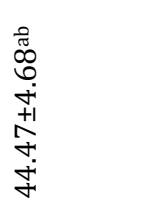 & 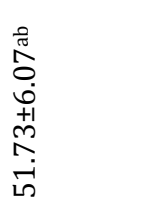 & 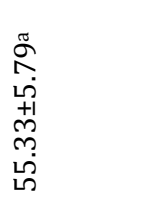 & 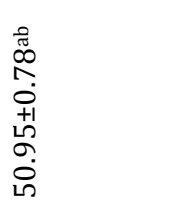 \\
\hline 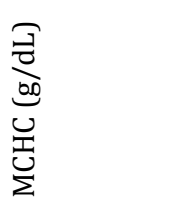 & 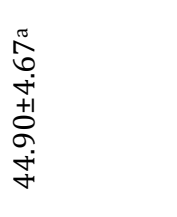 & $\begin{array}{l}\tilde{n} \\
\infty \\
\infty \\
\infty \\
+1 \\
+1 \\
0 \\
\infty \\
\infty \\
\infty\end{array}$ & 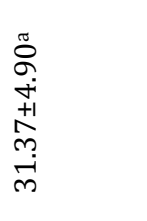 & 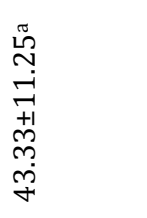 & 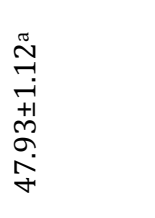 & 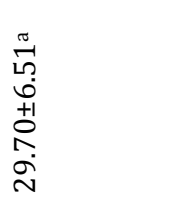 \\
\hline 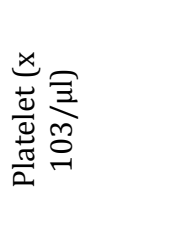 & 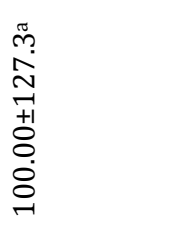 & 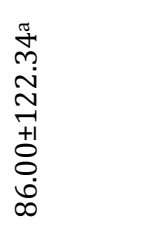 & 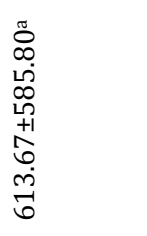 & 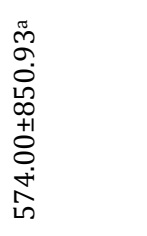 & 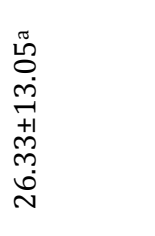 & 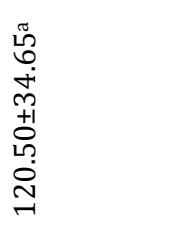 \\
\hline
\end{tabular}

Table 4: Haematological Assessment of Clarias Gariepinus Fed Varied Inclusion of DGNC Diet 
PCV=Packed Cell Volume; $W B C=$ White Blood Cell; Neut=Neutrophils; Lymph= Lymphocyte ; Mono=Monocytes;

Eosin=Eosinophil; Hb=Haemoglobin; RBC=Red Blood Cell; MCV= Mean Cell Volume; MCH=Mean Cell Haemoglobin;

\section{$\mathrm{MCHC}=$ Mean Corpuscular Haemoglobin}

However, microscopic examination of these organs revealed varying degrees of histological changes as a result of dietary treatments (Plates 1 to 18). Photomicrographs of sections of the gill of fish fed 0\% DGNC (control diet) show no visible lesion, there is atrophy of the lamellae in the gill of fish fed $20 \%, 60 \%$, and $80 \%$ DGNC (Plates 2, 4 and 5 ). There is fusion of the secondary lamellae in the gill of fish fed $40 \%$ (Figure 3). There is atrophy and fusion of secondary lamellae in the gill of C.gariepinus fed with 100\% DGNC (Figure 6). Photomicrograph of a section of the liver of C. gariepinus juveniles fed 0\% DGNC (control diet) showed no visible lesion (Figure 7). Fish fed 20\% DGNC shows moderate centrilobular cloudy swelling of hepatocytes (arrow) (Figure 8). The liver of fish fed 40\% DGNC had centrilobular hepatocellular vacuolar degeneration and necrosis (Figure 9). There is diffuse hepatocellular atrophy of the liver of C.gariepinus fed with $60 \%$ DGNC (Figure 10). There is centrilobular coagulation necrosis and vasculitis of the Liver of C.gariepinus fed with 80\% DGNC (Figure 11). Fish fed 100\% DGNC had centrilobular hepatocellular vacuolar degeneration of the liver (Figure 12). Photomicrographs of sections of the kidneys of fish fed $0 \%$ and $20 \%$ DGNC showed no visible lesions in their renal tubules (Plates 13 and 14). The kidneys of fish fed 40\% BSSM-based diet revealed patchy tubular epithelial coagulation necrosis (arrow) (Figure 15). The kidney sections of fish fed 60\% DGNC shows tubular atrophy and coagulation necrosis (arrow) (Figure 16). There was some patchy tubular epithelial coagulation necrosis in the kidney of C.gariepinus fish fed $80 \%$ and 100\% DGNC (Plates 17 and 18).

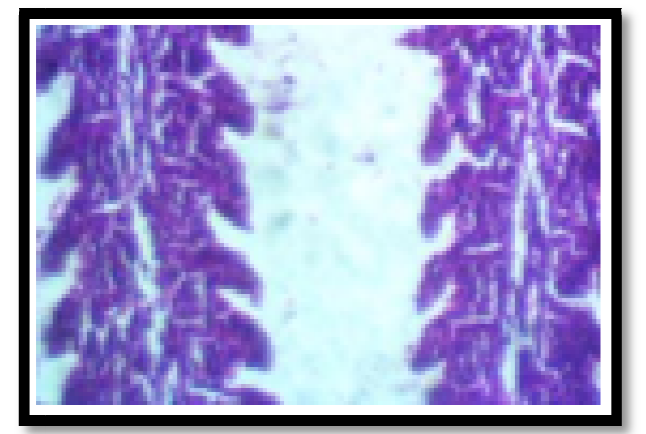

Figure 1: There Is No Observable Lesion in the Gill of C. Gariepinus Fed 0\% DGNC

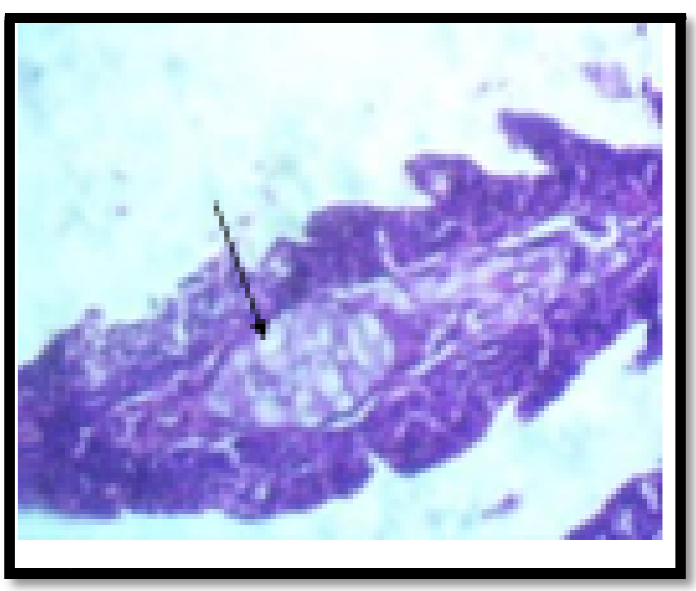

Figure 2: There Is Atrophy of Secondary Lamellae (Arrow) in the Gill of C.Gariepinus Fed 20\% DGNC. HE X400 


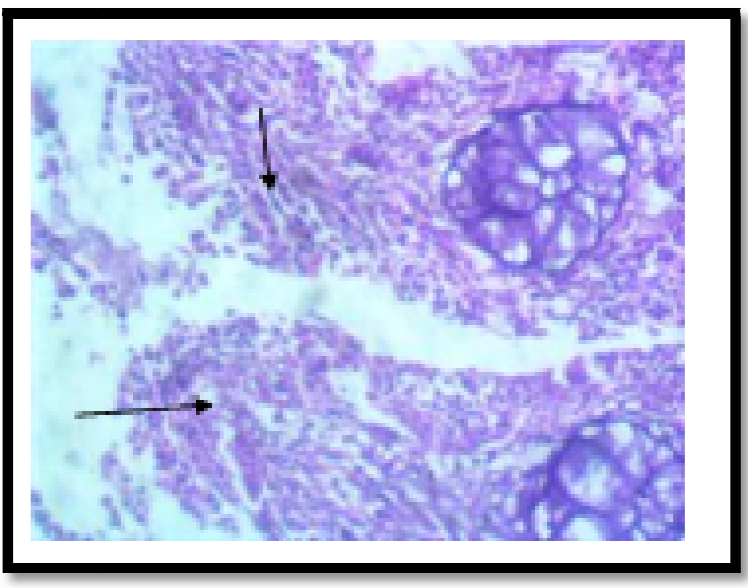

Figure 3: There Is Fusion (Arrow) of Secondary Lamellae in the Gill of C.Gariepinus Fed With 40\% DGNC, HE X400

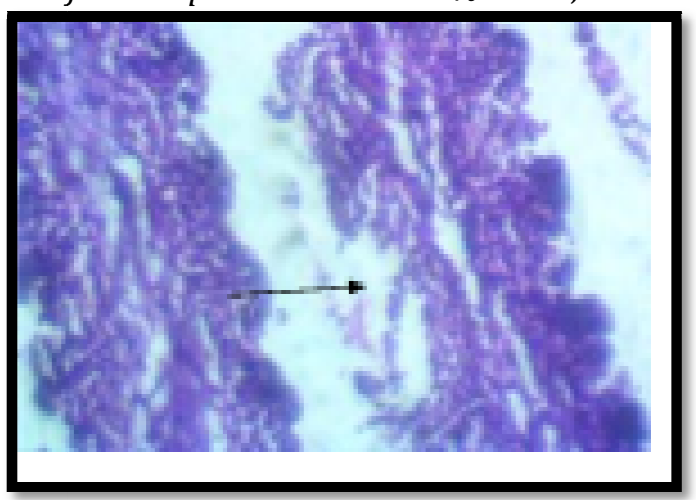

Figure 4: There Is Atrophy of Secondary Lamellae (Arrow) in the Gill of C.Gariepinus Fed with 60\% DGNC, HE X400

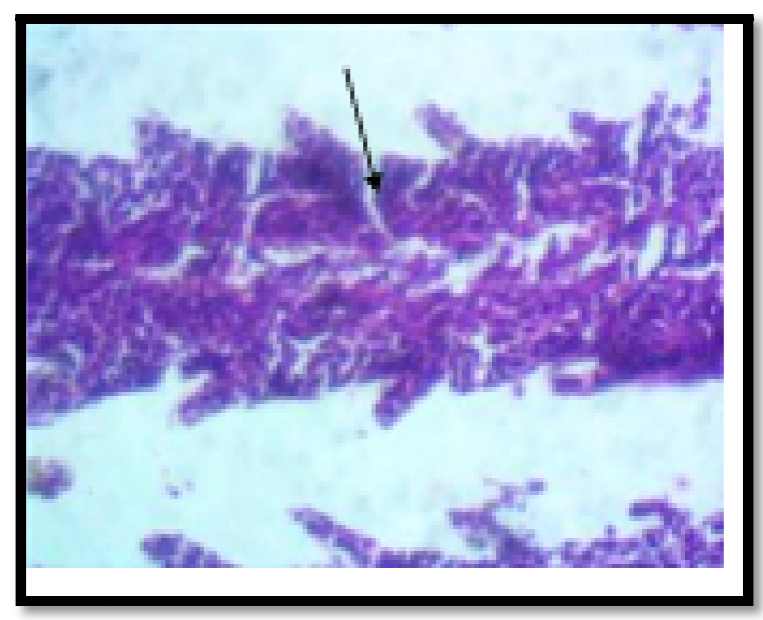

Figure 5: There Is Atrophy of Secondary Lamellae in the Gill of C.Gariepinusfed with $80 \%$ DGNC, HE X400

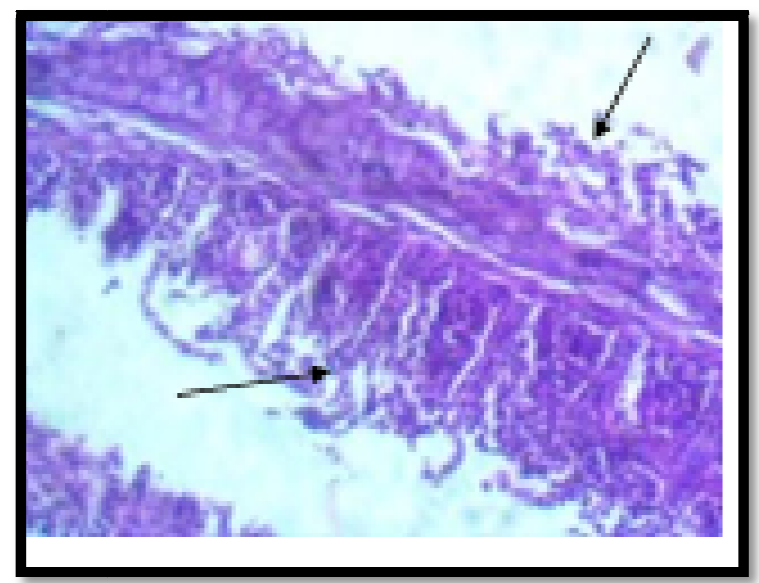


Figure 6: There Is Atrophy and Fusion of Secondary Lamellae in the Gill of C.Gariepinus Fed with 100\% DGNC, HE X400

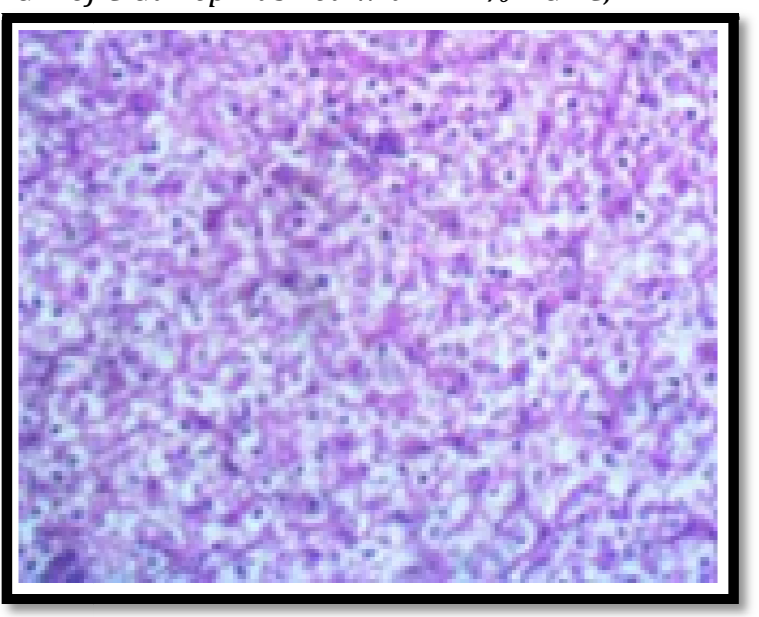

Figure 7: The Hepatocytes of the Liver of C.Gariepinus Fed with 0\% DGNC Are Vacuolated (Normal)

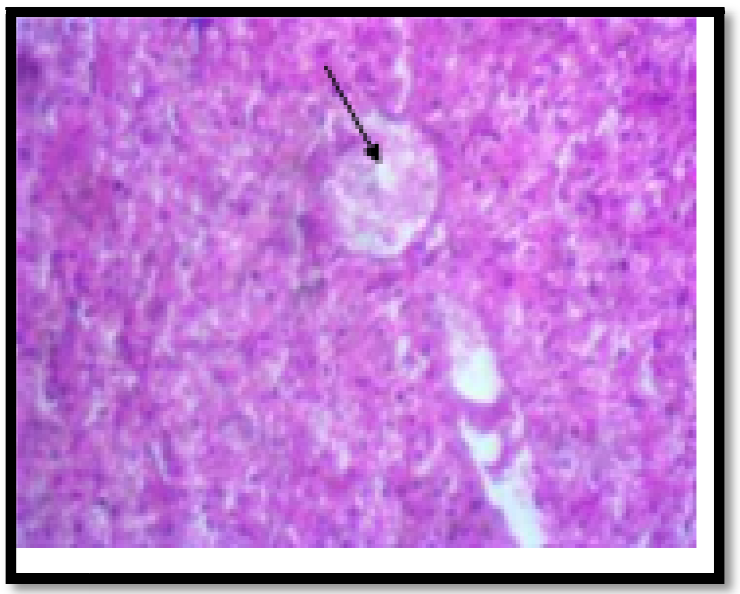

Figure 8: There Is Moderate Centrilobular Cloudy Swelling of Hepatocytes of C.Gariepinus with Fed 20\% DGNC, HE X400

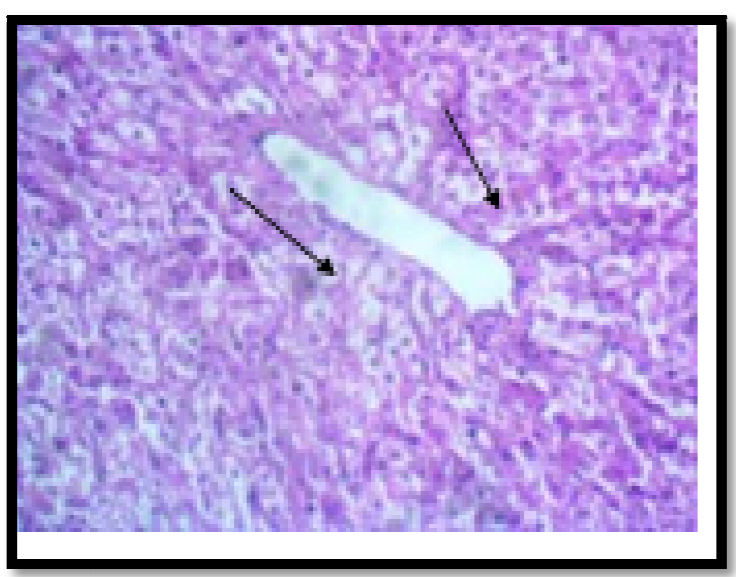

Figure 9: There Is Centrilobular Hepatocellular Vacuolar Degeneration and Necrosis of the Liver of C.Gariepinus Fed with 40\% DGNC (Arrows), HE X400 


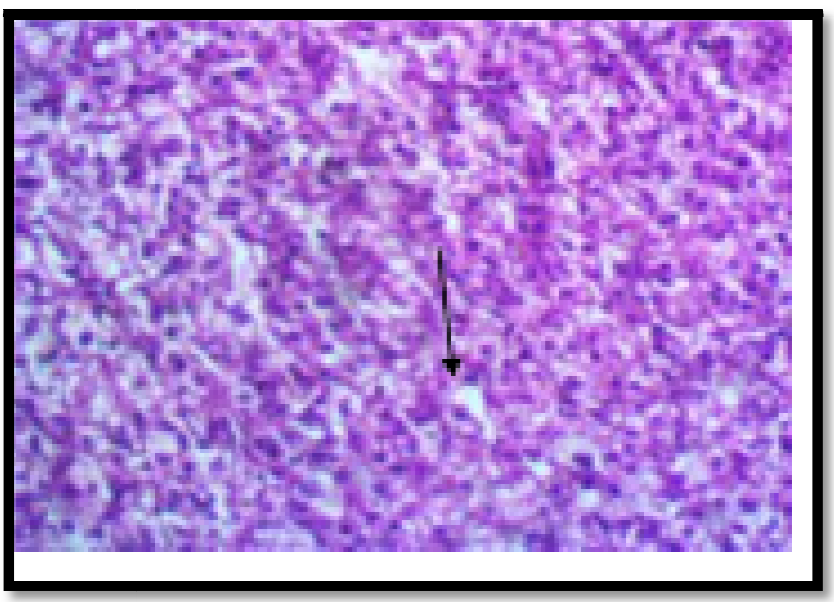

Figure 10: There Is Diffuse Hepatocellular Atrophy of the Liver Of C.Gariepinus Fed with 60\% DGNC (Arrows), HE X400

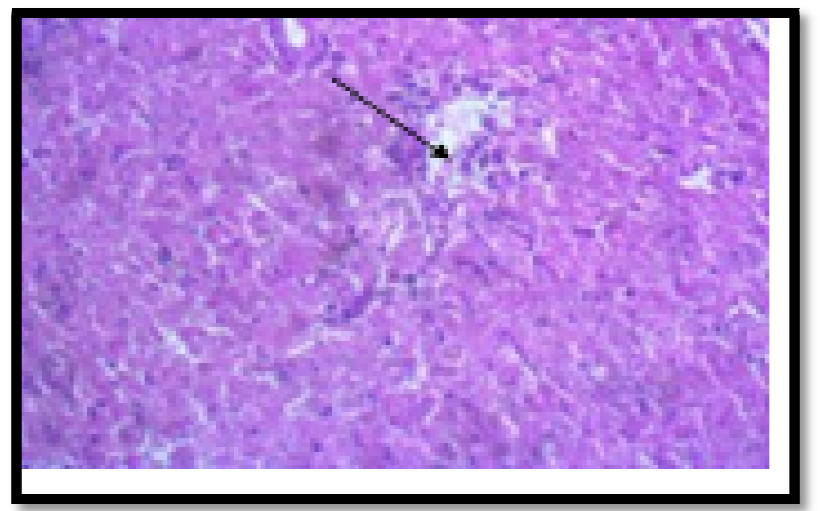

Figure 11: There Is Centrilobular Coagulation Necrosis and Vasculitis of the Liver OfC. Gariepinus Fed With 80\% DGNC, HE X400

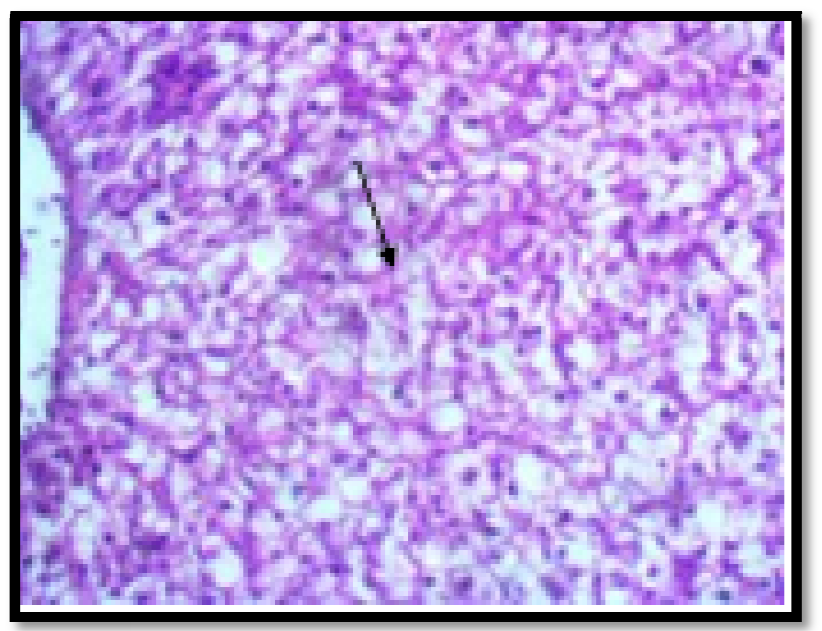

Figure 12: There Is Centrilobular Hepatocellular Vacuolar Degeneration of the Liver of C. Gariepinus Fed with 100\% DGNC, HE X400 


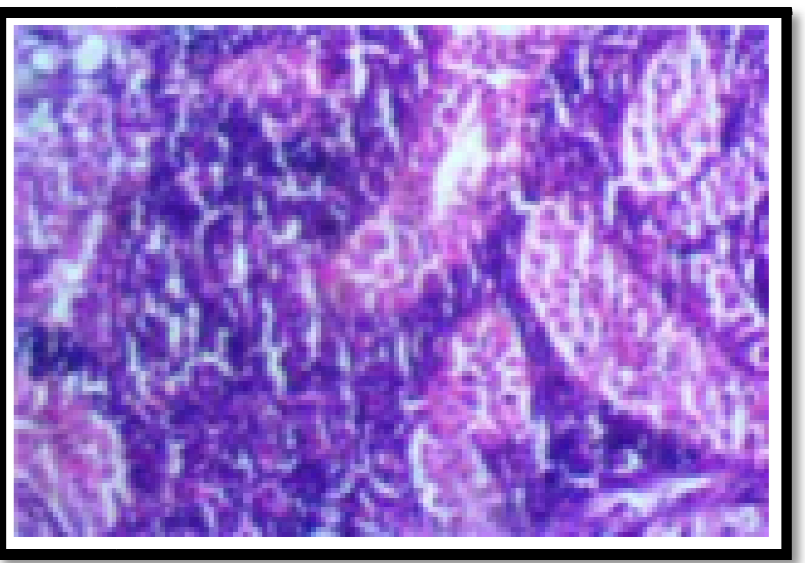

Figure 13: There Is No Observable Lesion in the Kidney of C.Gariepinus Fed with 0\% DGNC, Hex400

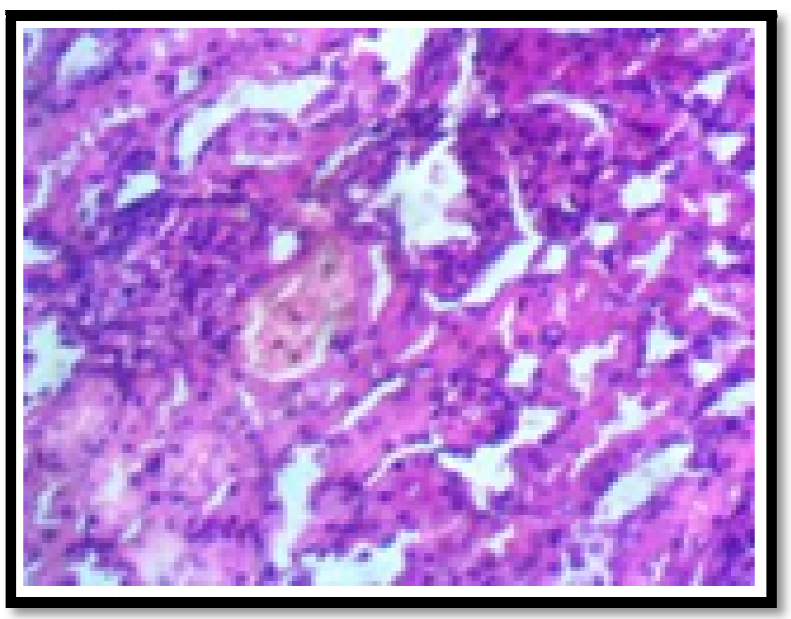

Figure 14: There Is No Observable Lesion in the Kidney of C.Gariepinus Fed with 20\% DGNC

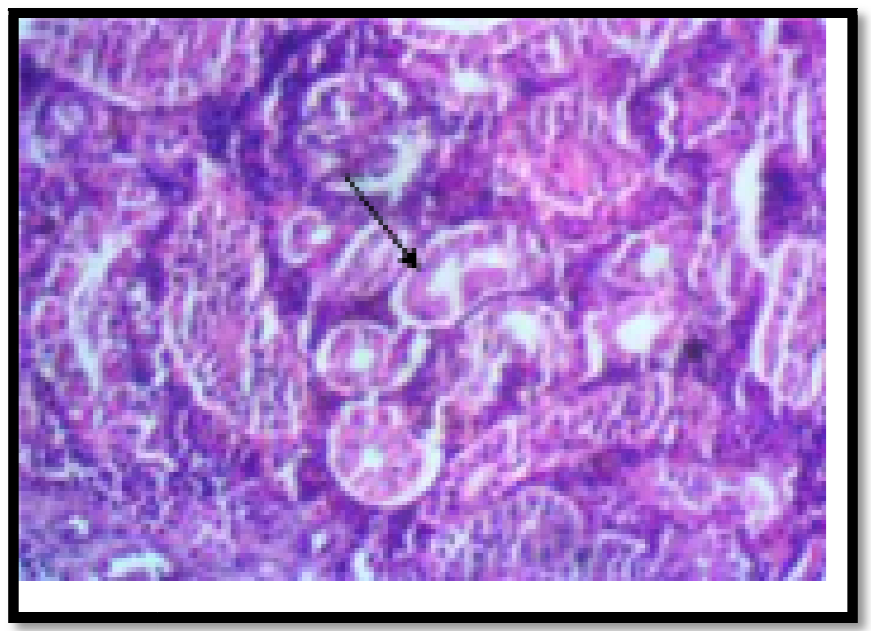

Figure 15: There Is Patchy Tubular Epithelial Coagulation Necrosis (Arrow) in the Kidney of C. Gariepinus Fed with 40\% DGNC, HE X400 


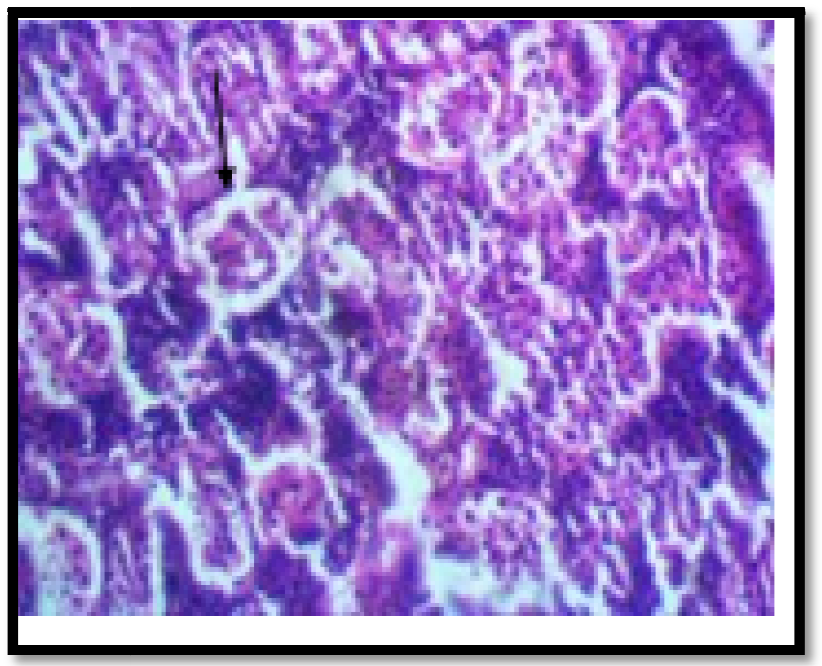

Figure 16: There Tubular Atrophy and Coagulation Necrosis (Arrow) In the Kidney of C.Gariepinus Fed with 60\% DGNC. HE X400

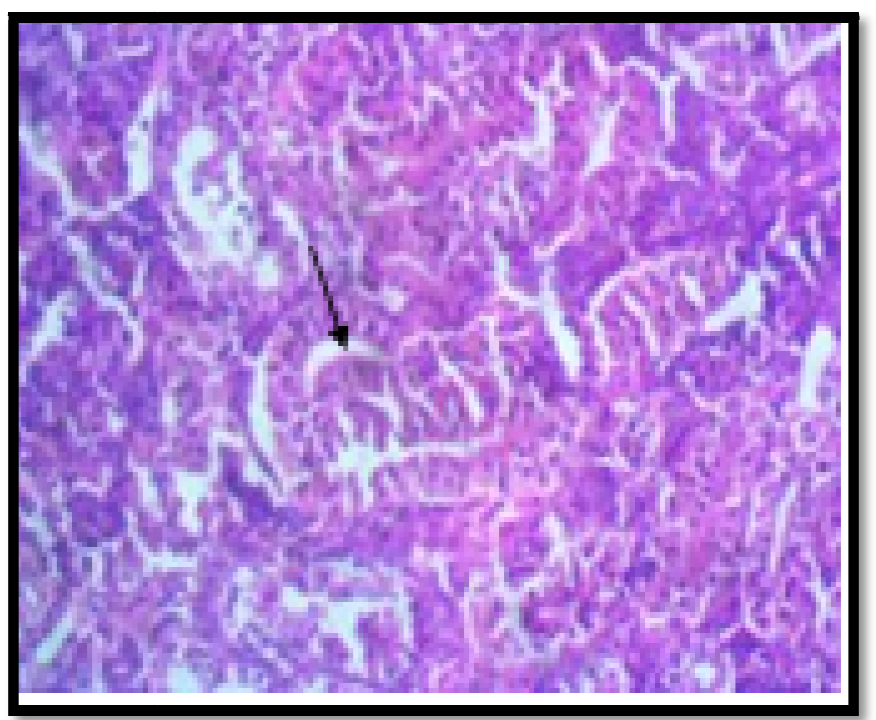

Figure 17: There Is Patchy Tubular Epithelial Coagulation Necrosis in the Kidney of C.Gariepinus Fed with 80\% DGNC, HE X400

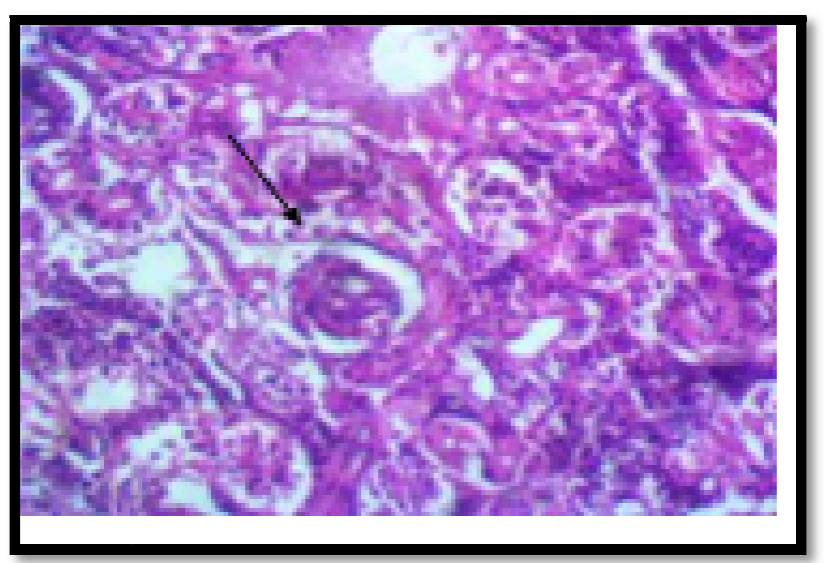

Figure 18: - There Is Patchy Tubular Epithelial Coagulation Necrosis in the Kidney of C.Gariepinus Fed with 100\% DGNC. HE X400

\section{Discussion}

The result obtained revealed that inclusion level of DGNC beyond 20\% inclusion level could results in decrease in growth rate of C. gariepinus juveniles. The growth and nutrient utilization by C. gariepinus juveniles decreased as the inclusion of DGNC increased in the diets. Similar findings were observed by Dienye et al., (2014) in C. gariepinus fed Moringa oleifera leaf meal. This reduction may be as a result of the high content of anti-nutrients such as cyanogenic glycosides, phytic acid and tannin present in the groundnut. Richter et al., (2003) showed that higher substitution of 
Moringa oleifera leaf meal with fish meal had an impact on lowering the growth performance because of the presence of anti-nutrients such as phenol, tannins, phytates and saponins.

There was reduction in the values of specific growth rate (SGR) of the experimental fishes with increasing level of DGNC in the diet. Hossain et al. (2003) and Adewolu (2008) reported that high inclusion of plant protein in the diets of C. gariepinus suppressed growth significantly. SGR values observed in this study didn't agreed with those reported by Erfanullah and Jafri (1998), Alegbeleye et al., (2001) and Ochang et al., (2007), who acknowledged that SGR of Clarias gariepinus post fingerlings fed control diet, were higher than those fed on diets containing leaf meals.

Protein efficiency ratio (PER) and feed conversion ratio (FCR) were best in fish fed with $20 \%$ inclusion level of DGNC diet. The reduction in growth observed in the other diets could be due to improper balance of essential nutrients such as amino acids, minerals and presence of some anti-nutritional compounds in the diet. Decrease in palatability and indigestibility due to high fibre content which may have led to reduced growth and poor food efficiency (Sogbeson and Ugwumba, 2006; Sotolu, 2008). These findings are similar to that of Chapman (2000) and Babalola and Apata (2006) who reported that the higher SGR and the lower feed conversion ratio (FCR).

The best survival rate $(97.50 \%)$ was observed in fish fed with the control diet. This result was against the findings of Farahi et al., (2012) who concluded that survival rate of fish was promoted in diets supplemented with Mellisa officinalis and Aloe vera. The highest protein intake (2.07) was obtained in fish fed 100\% (T5) inclusion of DGNC while the lowest value (1.36) was obtained in fish fed $20 \%$ (T2) inclusion of DGNC.

However, the fish fed 20\% DGNC showed overall good performance in terms of Final Mean Weight (FMW), Mean Weight Gain (MWG), Percentage Weight Gain (PWG), Specific Growth Rate (SGR), Feed Conversion Ratio (FCR), and Protein Efficiency Ratio (PER). Which could be attributed to low molecular weight of metabolites, improved palatability of the feed, and better digestibility of the feed and lower fibre content. This indicates that 20\% DGNC could replace soyabean meal in the diet of C. gariepinus fingerlings, juveniles and adults without any deleterious effect on the growth performance. Blood could be used as a means through which general condition of the animal body could be assessed. The PCV values in diet containing 100\% DGNC fell below the control but differ from other diets but other values were higher than the control value of $11.50 \pm 7.78$. However, Sotolu and Faturoti (2011) reported lower PCV values which were attributed to anaemia resulting from shrunken red blood cells, a situation that probably resulted in fish asphyxiation and death as confirmed by Adeyemo (2005). Increase in haematocrit is an indication of a stress response causing RBC swelling or haemoconcentration due to plasmatic volume reduction (Wilson and Taylor, 1993).

According to Ajani (2005) and Kori-Siakpere and Ubogu (2008), high WBC count means release of more cells to maintain homeostasis. Also, Douglas and Jane (2010) stated that the amount of the white blood cells has implication in immune responses and the ability of the animal to fight infection. There was an unusually increases in white blood cell count in all the diets with the highest value in diet containing 40\% DGNC with count of 57200.00 \pm 26972.39 (104/ mm3). Nordenson, (2004) stated that an unusually high WBC count might indicate hypersplenism, inflammation, trauma and stress. Ayoola (2011) further observed that there was an increase in the WBC of C. gariepinus fed poultry waste and attribute this to increase in the production of leucocytes in the haematopoietic tissue of the kidney and the spleen. The consequence of this is the suppression of the immune system and increased susceptibility to disease. Neutrophil in diet containing 100\% DGNC were significantly higher than the remaining diets. Foster (2011) reported that when total neutrophil increases it is a sign of bacterial infection or a form of stress. The cause of the increase in this study could not be ascertained but it is unlikely to be associated with bacterial infection as there was no sign of such infection in the fish used in the study.

The trend of Lymphocyte values for all the treatments indicate high Immune status of the experimental fish fed various experimental diet with the highest value of $96.67 \%$ observed in diet containing $20 \%$ DGNC. The lack of statistical variation between eosinophil values suggested that eosinophil was not affected by dietary treatments. Values of haemoglobin were highest diet containing 40\% DGNC (8.57 g/100 ml) and compared well with $8.70 \mathrm{~g} / 100 \mathrm{ml}$ for C. gariepinus (Sowunmi, 2003) as well as $8.90 \mathrm{~g} / 100 \mathrm{ml}$ reported by Dienye and Olumuji (2014). Church and Pond (1974) observed that haematological trait $\mathrm{Hb}$ values correlated with the nutritional status of the animal and the influence of diet on haematological trait is very strong. The decrease in RBC count in C. gariepinus fed DGNC in this study is similar to the observation of Omitoyin (2006) on the work haematological changes in the blood of C. gariepinus juvenile fed poultry waste. Ajani (2005) reported that low RBC values in extensive system is associated to stress due to high stocking and poor nutrient quality of feed also that low RBC is a common stress response.

The mean MCV values obtained in this study were lower than $240.18 f$ recorded for juvenile hybrid African catfish (Heteroclarias) reported by Kori-Siakpere and Ubogu (2008), $200.93 \mathrm{fl}$ for C. gariepinus fingerlings (Gbore et al., 2006). MCV as an estimate of the volume of RBCs indicates the status or size of the RBCs and reflects normal or abnormal cell division during RBC production (erythropoiesis). Larsson et al. (1985) attributed increase in MCV to swelling of the RBCs due to hypoxic condition (low oxygen condition), impaired water quality, somatic stress or macrocytic anaemia (swelling of RBCs) in fishes exposed to metal pollution. Reduced MCV could be linked with shrinkage of RBCs either due to hypoxia or microcytic anaemia (shrinkage of RBCs) as earlier reported by Bhagwant and Bhikajee (2000), Adesina (2008) and Alwan et al. (2009).

$\mathrm{MCH}$ values were higher than values obtained in earlier reports such as $24.24 \mathrm{pg}$ for C. gariepinus juveniles (Omitoyin, 2006), 33.10 pg for C. gariepinus (Ochang et al., 2007) and 51.50 pg for juvenile Heteroclarias (Gbore et al., 2006). The results of the present study also agreed with Olasunkanmi (2011) who reported a significant increase in the final $\mathrm{MCH}$ values in C. gariepinus fed raw mucuna seed meal-based diets. Higher MCH indicates a good volume of haemoglobin which indicates effective oxygen transportation in the bloodstream for healthy wellbeing of the fish (Diyaware et al., 2013). 
MCHC values were within the range recommended by Bhaskar and Rao (1989) for healthy fish and they closely agreed with $35.47 \mathrm{~g} / \mathrm{dL}$ recorded for Heteroclarias (Kori- Siakpere and Ubogu, 2008) and 33.67 to $39.03 \mathrm{~g} / \mathrm{dL}$ for juvenile inter-generic hybrid catfishes (Diyaware et al., 2013). Although MCHC values in diets containing $0 \%, 60 \%$ and $80 \%$ were significantly higher with the values of $44.90 \mathrm{~g} / \mathrm{dL}, 47.93 \mathrm{~g} / \mathrm{dL}$ and $43.33 \mathrm{~g} / \mathrm{dL}$ respectively. The variation between the present study and previous reports might be due to species differences and age of the fishes that greatly influence the values of haematological indices (Docan et al., 2010). Platelet counts were much higher than $175.92 \times 103 / \mu \mathrm{L}$ recorded for adult Sarotherodon melanotheron (Akinrotimi et al., 2007). The values were also greater than $19.25 \times 103 / \mu \mathrm{L}$ recorded for Clarias anguillaris (Diyaware et al., 2013). Platelets helps in diagnosing problems associated with bruising or hemorrhage.

Fish fed varying inclusion level of DGNC showed various histo-pathological changes in the gill, liver and kidney. These changes are indications of toxic effects of feeding varying inclusion level of DGNC to C. gariepinus since no histological change was observed in the diet containing $0 \%$ DGNC This observation is in agreement with the report of Iyayi et al. (2005) who reported that broiler birds fed heated and raw Mucuna bean meals produced varied histological signs in their organs.

The gill in the diets containing 0\% DGNC had normal morphology of the hyaline cartilaginous rods in each filament, while the gill of fish fed 20\%, 40\%, 60\%, 80\% and 100\% DGNC showed histological changes which included atrophy of the secondary lamellae, fusion of the secondary lamellae, atrophy and fusion of secondary lamellae. According to Fafioye et al. (2004), these histopathological changes in the gills are similar to epithelial damages caused by cadmium. The alterations produced may probably lead to several physiological stresses in the fish.

The histopathology of the liver of fish fed with 0\% DGNC showed that the liver was normal, feeding varying inclusion level of 20\%- 100\% DGNC to C. gariepinus resulted to various lesions in the liver cells of the fish. The lesions ranged from moderate centriobular cloudy swelling of hepatocytes to centrilobular hepatocellular vacuolar degeneration and necrosis, to centriobular coagulation necrosis and vasculitis and centriobular hepatocellular vacuolar degeneration. This observation agrees with the work of Jha (2004) who reported remarkable lesions in the liver of C. batrachus exposed to surf and that of Ayoola (2008) on the effects of glyphosate in C. gariepinus. Omitoyin et al. (2006) also reported similar observation in fish exposed to Lindane. Ukachukwu et al. (2003) reported that diets containing raw beans caused wide area of periportal necrosis with some mononuclear cell infiltration in the liver of broilers, while the centrilobular areas showed vacuolation and degeneration of hepatocytes. Olasunkanmi (2015) also observed that C. gariepinus fed processed velvet beans showed both mild diffuse and marked widespread vacuolation of the hepatocytes. Vacuolated hepatocytes are usually accumulated with glycogen and have little or no regenerative ability (Nayak et al., 1996) and the excessive vacuolation of the hepatocytes would result in abnormal functioning of the liver cells, Ayoola (2008) opined that vacuolation of liver cells is an evidence of fatty degeneration. In the present study, lesions observed in the liver may probably have resulted from the excessive work done by the fish to get rid of toxicants from its body during the process of detoxification by the liver. Hepatocytes in the periportal areas suffer most from toxic insults. In this situation, toxic substances present in Mucuna beans must have been responsible for the histopathological changes found in the liver sections.

The result of the present study closely supports the finding of Hlophe and Moyo (2014) who observed that C. gariepinus fed high moringa leaf meal inclusion levels ( $>50 \%$ ) showed an increase in the number of degraded irregularly shaped hepatocytes, small dark pyknotic nuclei, poor fatty deposition and isolated necrosis. Despite similar protein and energy levels in the experimental diets, liver histology showed that fish fed higher DGNC inclusion levels had necrotic signs associated with poor nutritional status (Tusche et al., 2012). The malnutrition signs observed in C. gariepinus fed higher levels of DGNC might be due to non-availability of protein and amino acids that have bound with or have formed indigestible complexes with the anti-nutritional compounds in the groundnut cake meal. As a result of the poor digestibility, a substantial portion of the essential dietary nutrients was not available to the fish and was subsequently excreted. This explains the nutritional necrosis observed in the hepatocytes.

Utilization of varying inclusion level of DGNC by C. gariepinus also resulted in some histological changes in its kidney which included patchy tubular epithelial coagulation necrosis, tubular atrophy and coagulation necrosis, Olasunkanmi (2015) also observed a marked congestion in the kidneys of C. gariepinus juveniles fed diets containing higher inclusion levels of processed velvet beans and associated such changes with ingestion of a high percentage of velvet bean meals which imposed stress on the organ above its physiological capacity. Benjamin (2009) earlier reported that congestion of the kidney tubules is the first stage in the development of kidney disease.

Most common alterations found in the kidneys of fishes are tubule degeneration, dilation of capillaries in the glomerulus and reduction of Bowman's capsular space (Takashima and Hibya, 1995). The presence of tubule disruption and necrosis in the kidney of fish indicates that the kidney suffered some damage which could be attributed to the presence of anti-nutritional substances in the diet

\section{Conclusion}

Fish fed with up to $20 \%$ defatted groundnut cake grew fairly well compared to the other experimental diets, inclusion of defatted groundnut cake beyond this level can led to comparatively low weight gain. The best haematological parameters were observed at the inclusion level of $40 \%$ followed by $20 \%$ which is in correlation with the $20 \%$ inclusion level for growth parameters. While inclusion level above $20 \%$ shows that the organs were severely impaired. It is therefore very harmful to the health of the fish to substitute beyond this level. 


\section{References}

i. Adewolu, M. A., (2008). Potentials of sweet potato (Ipomoea batatas) leaf meal as dietary ingredient for Tilapia Zilli fingerlings. Pakistan Journal of Nutrition 7(3): 444-449.

ii. Adeyemo, O. K. (2005). Haematological and Histopathological effects of cassava mill effluent in Clarias gariepinus. African Journal of Biomedical Research, 8: 179-183.

iii. Akinrotimi, O. A., U. U. Gabriel, P. E. Anyanwu and A. O. Anyanwu. (2007). Influence of sex, acclimation methods and period on haematology of Sarotherodon. melanotheron. Research Journal of Biological Science, 2: 348-352.

iv. Alegbeleye, W.O., Oresequn, A. and Ajitomi, 0.0. (2001). An Assessment of Jack bean (Canavalia ensiformis) Meal as an Ingredient in the Diets for Clarias gariepinus (Buchell, 1822) fingerlings. Fish Nutrition and Feed Technology, Edited by Eyo, A.A., FISON, pp. 92 -97.

v. Alwan, S.F., A.A. Hadi and A.E. Shokr. (2009). Alterations in Haematological Parameters of Fresh Water Fish, Tilapia zillii, Exposed to Aluminum. Journal of Science, 3 (1): 12- 19.

vi. A.O.A.C. (Association of Official Analytical Chemists), (2006). Official methods of Analysis of the AOAC, W. Horwitz Editor Eighteen, 380pp. Washington D.C., U.S.A.

vii. Ayoola, S.O. (2011) Haematological Characteristics of Clarias gariepinus (Buchell, 1822) Juveniles Fed with Poultry Hatchery Waste. Iranica Journal of Energy \& Environment 2 (1): 18-23.

viii. Babalola, T. O. and D. F. Apata (2006). Effects of dietary protein and lipid levels on the growth performance and body composition of African catfish, Heterobranchus longifilis fingerlings. Journal Animal Veterinary Advances,8(5):1073-1079.

ix. Barbieri, E.; Bondioli, A. C. V. (2015) Acute toxicity of ammonia in Pacu fish (Piaractus mesopotamicus, Holmberg, 1887) at different temperatures levels. Aquaculture Research, 46(3): 565-571.

x. Barros, M. M.; Ranzani-Paiva, M. J. T.; Pezzato, L. E.; Falcon, D. R.; Guimarães, I. G. (2009) Hematological response and growth performance of Nile tilapia fed diets containing folic acid. Aquaculture Research, 40(8): 895-903.

xi. Benjamin, H. (2009). Diseases of the liver, gall bladder, kidneys and pancreas. Accessed online from www: herbdatanz.com/liver gall bladder kidney pancreas harry Benjamin nd.htm on 21/05/2011.

xii. Bhagwant, S. and M. Bhikajee. (2000). Induction of hypochromic macrocytic anaemia in Oreochromis hybrid (Cichlidae) exposed to 100mg/l (sublethal dose) of aluminum. Journal of Science and Technology, 5: 10-20.

xiii. Chapman, F. A. (2000). Farm raised catfish. Department of fisheries and aquatic sciences, Florida cooperative Extension service. Institute of Food and Agriculture Sciences, University of Florida. Circular No. 1052.

xiv. Dacie, J. V. and Lewis. S. M. 1991. Practical Hematology. Churchill Livingstone, London.

xv. Davies, O. A. and N. C. Ezenwa, (2010). Groundnut cake as alternative protein source in the diet of Clarias gariepinus fry. International journal of science, 1: 73-76.

xvi. Dienye, H. E. and O. K. Olumuji. (2014). Growth performance and haematological responses of African mud catfish Clarias gariepinus fed dietary levels of Moringa oleifera leaf meal. Net Journal of Agricultural Science, 2 (2): 79-88.

xvii. Diyaware, M. Y., Haruna A. B., Abubakar K. A. (2013). Some haematological parameters of intergeneric hybrid of African catfish (Clarias anguillaris $\times$ Heterobranchus bidorsalis) juveniles and their pure lines in north-eastern Nigeria. Journal of Fisheries and Aquatic Science, 8 (1): 33-42.

xviii. Docan, A., V. Cristea, L. Grecu and L. Dediu. (2010). Haematological response of the European catfish, Silurus glarus reared at different densities in flow-through production system. Archive Zootechnica, 13 (2): 63-70.

xix. Douglas, J. W. and K. W. Jane. (2010). In Schalm's Veterinary Haematology. John Wiley and Sons. Blackwell publishing Ltd. pp: 1232.

xx. Erfanullah and Jafri, A.K. (1998). Growth Response, Feed Utilization and Nutrient Retention in Catla cattle (Ham.) Fry Fed Varying Levels of Dietary Carbohydrate. Asian Fisheries Science, 11:223 - 230.

xxi. Eyo, A. A. and Olatunde, A. A. (1998) Effect of supplementation of soya bean diet with L and L- methionine on the growth of mudfish C. auguillaris fingerlings. Nigeria. Journal of Biotechnology, 9 (1): 9- 16.

xxii. Fafioye, 0.0. (2001) Lethal and Sub-lethal Effects of extracts of (Parkia biglobossa) and (Raphia vinifera) extracts on (Clarias gariepinus) on some freshwater Fauna PhD Thesis, University of Ibadan, Nigeria. 216p.

xxiii. FAO (2000) NACA/FAO Report of the Conference on Aquaculture in the third millennium, Bangok, Thailand, 20 - 25 February, 2000. FAO Fisheries Report N0. 661. Rome. 97p.

xxiv. Farahi A., Kasiri M., Sudager M., Soleiman I.M., and Zorrieh-Zahra S.M.J. (2012). Effect of Dietary Supplementation of Mellisa officinalis and Aloe vera on Haematological Traits, Lipid Oxidation of Carcass and Performance in Rainbow Trout (Oncorhynchus mykiss). Journal of Animal and Feed Research. 2(1):01-05.

xxv. Gbore, F. A., O. Oginni, A. M. Adewole and J. O. Aladenton. (2006). The effect of transportation and handling stress on haematology and plasma biochemistry in fingerlings of Clarias gariepinus and Tilapia zillii. World Journal of Agricultural Science, 2: 208-212.

xxvi. Halver, J.E. (1972). The vitamins. In: Fish nutrition, edited by J.E. Halver. New York, Academic Press, pp. $29-103$.

xxvii. Hlophe, S. N. and Moyo, N. A. G. (2014). Replacing Fishmeal with Kikuyu Grass and Moringa Leaves: Effects on Growth, Protein Digestibility, Histological and Haematological Parameters in Clarias gariepinus. Turkish Journal of Fisheries and Aquatic Sciences 14: 795-806.

xxviii. Iyayi, E. A., Taiwo V. O. and Fagbohun A. O. (2005) Performance, Carcass Characteristics, Haematological and Histopathological Studies of Broilers Fed Mucuna (Mucuna Utilis) Bean Meal Based Diets. Israel Journal of Veterinary Medicine, 60, (2): 51- 57. 
xxix. Jha, B. S. (2004) Toxicological Impact of Household detergent, Surf, on Digestive Tissues of the Freshwater Fish, (Clarias batrachus) Fish Research: Vision for 21st Century edited by B. N. Pandey. A. P. H. Publishing Corporation, New Delhi.

xxx. Joshi P. K, Bose M and Harish D (2002) Haematological changes in the blood of Clarias batrachus exposed to mercuric chloride. Ecotoxicological Environmental Monitoring 12: 119-122.

xxxi. Kori-Siakpere, O. and E. O. Ubogu. (2008). Sublethal haematological effects of zinc on the freshwater fish, Heteroclarias sp. (Osteichthyes: Clariidae). Journal of Biotechnology, 7 (12): 2068-2073.

xxxii. Nayak, N. C., Sathar, S. A., Mughal, S., Duttagupta, S., Mathur, M. and Chopra, P. (1996). The nature and significance of liver cell vacuolation following hepatocellular injury - an analysis based on observations on rats rendered tolerant to hepatotoxic damage. Virchows Archives 428: 353-365.

xxxiii. Ochang, S.N. Fagbenro, O.A. and Adebayo, O.T. (2007). Growth Performance, Body Composition, Haematology and Product Quality of the African Catfish (Clarias gariepinus) Fed Diets with Palm Oil. Pakistan Journal of Nutrition, 6(5): $452-459$.

xxxiv. Olapade Olufemi Julius and George Patrick Quinn, (2019). Nutritional evaluation of defatted groundnut cake meal with amino acid as protein supplement in African catfish (Clarias gariepinus Burchell, 1822) juveniles' diet. Journal of Fish Aquaculture Science, 14: 7-14.

xxxv. Olasunkanmi, J. B. (2011). Nutrient utilization and growth performance of Clarias gariepinus fed differently processed Mucuna utilis meals as a replacement for soybean-based diet. PhD. Thesis, University of Ibadan, Ibadan, Nigeria. 209pp

xxxvi. Omitoyin, B. O. (2006). Haematological changes in the blood of Clarias gariepinus (Burchell1822) juvenile fed poultry litter. Livestock Research for Rural Development, 18 (11):1- 6.

xxxvii. Richard D. M. and Chapman F. A. (2007). The concept of ideal protein in formulation of Aquaculture feeds. NAGA World Fish Center Quarterly Report Vol. 26. No 3.

xxxviii. Samuelson A (2007) Textbook of veterinary histology. Elsevier Health Sciences Publishing Company, London, United Kingdom. 560 pp.

xxxix. Siddhuraju, P. and Becker, K. 2001. Preliminary nutritional evaluation of mucuna seed meal (Mucuna pruriens var. utilis) in common carp (Cyprinus carpio L.): An assessment by growth performance and feed utilization. Aquaculture 196:105-123.

xl. Sintayehu, A., Mathies, E., Meyer-Burgdorff, K. H., Rosenow, H. and Gunther, K.-D. (1996). Apparent digestibility and growth experiments with tilapia (Oreochromis niloticus) fed soybean, cottonseed meal and sunflower seed meal. Journal of Applied Sciences, 12 (2): 125-130.

xli. Sogbesan, O. A., Ugwumba, A. A. A. and Madu, C. T. (2006). Nutritive potential and utilization of garden snail (Limicolaria aurota) meat in the diet of Clarias gariepinus fingerlings. African Journal of Biotechnology, 5(20):1999-2003.

xlii. Sotolu, A. 0. (2008). Nutrient potential of water hyacinth as a feed supplement in sustainable aquaculture. Obeche 26, (1): 45-51.

xliii. Sotolu, A. O. and E. O. Faturoti. (2011). Digestibility and Nutritional Values of Differently Processed Leucaena leucocephala (Lam De Wit) Seed Meals in the Diet of African Catfish (Clarias gariepinus). Middle-East Journal of Scientific Research, 3 (4): $190-199$.

xliv. Sowunmi, A. A. (2003). Haematology of the African catfish, Clarias gariepinus (Burchell 1822) from Eleyele reservoir, Ibadan, Southwest Nigeria. The Zoologist, 2 (1): 40-44.

xlv. Stickey, R. R., Hardy, R. W., Koch, K., Harold, R., Searight, D. and Massee, K. C., Tacon, A. G. J., and Jackson, A. J. (2006). Utilization of conventional protein sources in practical fish feeds. Journal of Food Science 2(4): 45-56.

xlvi. Stoskopf, M. K. (1993). Clinical pathology in fish medicine. W.B. Saunders Company, Hartcourt Brace Jovanourah Inc.

xlvii. Takashima, F. \& T. Hibiya. 1995. An atlas of fish histology: normal and pathological features. 2nd ed.Tokyo, Kodansha Ltda/ Stuttgart, Gustav Fischer Verlag, 213p.

xlviii. Thophon, S., Kruatrachue, M., Upathan, E. S., Pokethitiyook, P., Sahaphong, S. and Jarikhuan, S. (2003). Histopathological alterations of white seabass, Lates calcarifer,in acute and sub-chronic cadmium exposure. Environmental Pollution 121:307-320.

xlix. Tusche, K., Arning, S., Wuertz, S., Susenbeth, A. and Schulz, C (2012) Wheat gluten and potato protein concentrate - Promising protein sources for organic farming of rainbow trout (Oncorhynchus mykiss). Aquaculture, 349: 120-125.

l. Ukachukwu, S. N., Shoyinka, V. O. And Obioha, F. C. (2003) Chronic toxicity of raw lyon"s bean (Mucuna cochinchinensis) in broilers [toxicidad crónica del frijol lyon (Mucunacocnhinchinensis) pollos de engorda]. Tropical and Subtropical Agroecosystems, 2 (2003): 23 -30. 